\title{
Cities at War
}



Cities at

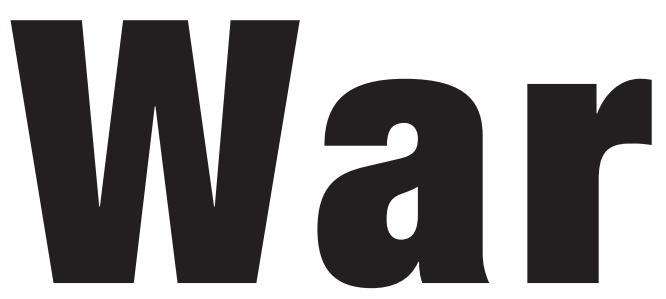

\section{Global Insecurity and Urban Resistance}

Mary Kaldor

\section{Saskia Sassen}

\section{EDITORS}

Columbia University Press Nerw York 
Columbia University Press

Publishers Since I893

New York Chichester, West Sussex

cup.columbia.edu

Copyright (C) 2020 Columbia University Press

All rights reserved

Library of Congress Cataloging-in-Publication Data

Names: Kaldor, Mary, author. | Sassen, Saskia, author.

Title: Cities at war : global insecurity and urban resistance / edited by Mary K. Kaldor and Saskia Sassen.

Other titles: Global insecurity and urban resistance

Description: New York : Columbia University Press, [2020] |

Includes bibliographical references and index.

Identifiers: LCCN 2019035766 | ISBN 9780231185387 (cloth) |

ISBN 9780231185394 (pbk.) | ISBN 9780231546133 (e-book)

Subjects: LCSH: Urban warfare-Developing countries-Case studies. |

Internal security-Developing countries-Case studies. | Metropolitan areas-Developing countries-Strategic aspects. | Urban violenceDeveloping countries—Case studies. | Political violence-Developing countries-Case studies. | Low-intensity conflicts (Military science)— Developing countries. | Human security—Developing countriesCase studies. | Sociology, Urban.

Classification: LCC Ui67.5.S7 K 35 2020 | DDC 355.4/26—dc23

LC record available at https://lccn.loc.gov/2019035766

Columbia University Press books are printed on permanent and durable acid-free paper.

Printed in the United States of America

Cover design: Lisa Hamm 